\title{
Towards a Conceptual Model of Talking to a Route Planner
}

\author{
Stephan Winter and Yunhui Wu \\ Department of Geomatics, The University of Melbourne, Victoria 3010, Australia \\ winter@unimelb.edu.au, y.wu21@pgrad.unimelb.edu.au
}

\begin{abstract}
Imagine a (web-based or mobile) route planning service that understands and behaves like another person. This conceptual paper addresses the first step towards this vision. It looks at the ways people would like to talk to their route planner in the initial phase of the route communication when specifying the travel route and time. The paper systematically collects service requirements, based on elements from intelligent autonomous agents, and demonstrates that a fundamental change is required compared to how services operate today.
\end{abstract}

Keywords. web-based route planning, human computer interface, ontologies, place.

\section{Introduction}

This paper studies the common question of a person: "Can you tell me the way to ...", which initiates a typical everyday communication on routes, either with other persons, or with dedicated route planning services ${ }^{1}$. Despite of its commonality this question forms quite a challenge for route planning services. Looking at current services' apparently simple interfaces-Figure 1 presents a typical subset of current route planners-it appears that they fail to accept above's question as is, in its context and with all kinds of location references a person may have in mind.

In another paper we have proposed a spatial Turing test for route planning services, postulating that services' behavior should aim to become indistinguishable from human-generated route advice to overcome obstacles in their usage [53]. Going back in that paper to Artificial Intelligence (in particular to [48]) was motivated by sharing the general interests: On one hand the scientific interest in gaining knowledge of human cognitive processes, and on the other hand the scientific interest in implementing formal models of these processes in machines. It was also motivated by the observation that communication with other people is more successful than with route planning services:

\footnotetext{
${ }^{1}$ For the purpose of this paper we include route planners of any form: web-based (desktop) or wireless (mobile), local or centralized, off-line (planning before traveling) or on-line (accompanying the travel), and even printed ones (e.g., time tables), believing that the architecture is relatively unimportant for our interest and also not always transparent to the wayfinder. We also include route planners for any mode of traveling such as walking, cycling, driving, or riding on public transport, trains or planes, but believe that in the future route planners have to integrate modes of traveling.
} 


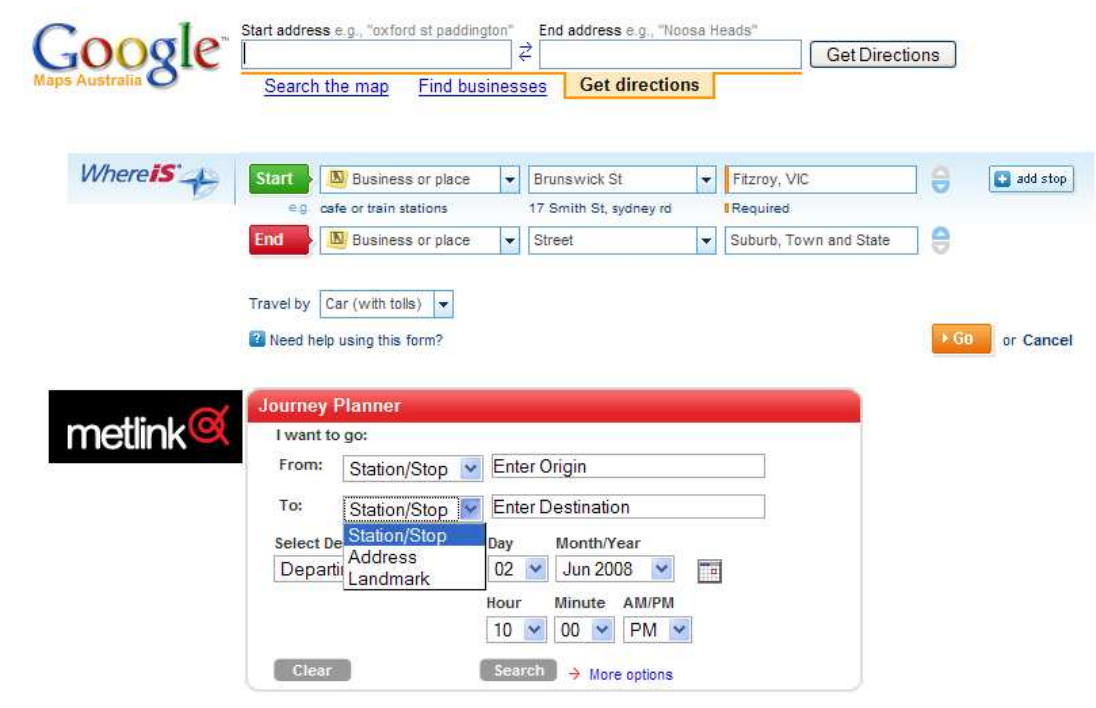

Fig. 1. The initial phase of a communication with different route planners (Google Maps, WhereIs, and Melbourne's public transport planner metlink).

- People have a richer spatial language, compared to the rigid syntax, grammar and ontology of route planners. But when talking to a route planning service, the interface requires the wayfinder to adapt to a restricted, rigid language (e.g., postal addresses, see Figure 1).

- People have more compatible spatial concepts and conceptualizations with each other, compared to the concepts provided by route planners. The concepts behind a route planner's interface are often determined by the service's internal needs, not by wayfinder needs (e.g., stop names of a bus network are difficult be known by wayfinders, see Figure 1).

- People have superior capabilities to capture a communication context and to adapt information to this context They communicate two-ways. Route planners have implemented this strategy only rudimentary (e.g., when resolving ambiguities by showing lists of alternatives) and cannot adapt.

Thus, route planners need to share some of the visions of Artificial Intelligence. They need to understand and support an autonomous and intelligent agent-the human wayfinder-although they are not autonomous by themselves. This means they should be able to cope with the characteristics and capabilities of an intelligent agent, which Brooks [1] has categorized as situatedness, embodiment and emergence. This paper will study the consequences of this postulate.

Winter and $\mathrm{Wu}[53]$ have looked at the complete dialog between a wayfinder and a route planner, from addressing the route planner to closing the communication. In contrast, the present paper will go a step further and focus on one element only, the initial phase where the wayfinder talks to the route planner to request a route. To illustrate 
anthropomorphic behavior in this phase let us consider the case of Mary. When one morning Mary jumps into her shoes to leave her apartment she would like to tell her device: "To uni!", and this device would tell her that she is in time to get the tram. This means the device would have succeeded to capture the context (from here and now, and by public transport is at least an option) and to match the colloquial and ambiguous location uni. Mary could also have asked her housemate sitting at her laptop about the way to the university: "Can you check my way to uni, please?" Well, the tone is different, but this question conveys the same content, and Mary's housemate would have had no problems either to understand her. But Mary, having an intelligent device-in Turing's sense of a device with anthropomorphic behavior-would not need to interrupt her housemate in her work.

This paper aims to develop the requirements of an anthropomorphic behavior for a service in the initial phase of a route communication. The hypothesis is that the requirements lead to a fundamentally different wayfinder interface compared to current route planner interfaces. If this is the case then route planning services cannot evolve into intelligent services; they require a paradigm shift in their internal design. To deal with the hypothesis we will study the three properties of an intelligent wayfinder: situatedness, embodiment and emergence. This will allow us to analyze the requirements of a service capable to understand their users.

To this extent this paper is purely conceptual, backed by research in spatial cognition. An experimental verification of the conceptual model by an implementation is beyond the scope of this paper and left for future work. Also left for future work are the consequences of this conceptual model for the other phases of the communication about routes.

The paper starts with a review of recent work (Section 2) to introduce the spatial Turing test. Then it studies the structure and tasks of the communication with a route planning service, in particular to identify the initial phase in which the wayfinder talks to the route planner (Section 3). Finally it embarks on the requirements analysis (Section 4) based on the desired characteristics identified in the previous section. The list of requirements is then discussed for the complexity of the challenge they pose (Section 5). This discussion shows strong evidence for the hypothesis. At the end the paper develops future research questions based on the requirements (Section 6).

\section{Background}

The Turing test of whether machines can think is an imitation game: a computer has to convince players that they are communicating with a person [48]. By this way Turing avoids to define thinking or intelligence; in Turing's sense a machine is intelligent if it shows anthropomorphic communication qualities.

We know that people reason on a hierarchical mental representation of space [17], which for instance leads to efficient communication about place [46]. We also know that a person's landmark-based, multi-granular route description is cognitively more efficient than a geometric route description as produced by current route planners. Human spatial representations are rather qualitative than geometric, and human communication of route directions nearly never refers to geometry in a metric manner $[7,45]$ because 
people have difficulties to realize the metrics. Cognitive efficiency can be shown for example by navigational performance [5]. Research demonstrates that cognitively ergonomic route directions (for example, as given by persons) have some redundancy [32], have varying, if not asymmetric granularity [21,46,36], relate to the perceptual experience of the environment [6], and refer to a selected range of features depending on the traveling mode [24,2]. Current route planners cannot yet imitate persons in these ways. Accordingly, one aim of current research is generating landmark-based route descriptions automatically $[31,33,12,15,46]$, which is surprisingly complex to realize in implementations.

Human route communication is yet superior to current services' route communication with respect to the cognitive effort of understanding, memorizing and realizing spatial descriptions in orientation and wayfinding. Hence, the spatial Turing test sets the bar for route planning services. Human communication has qualities challenging computers, such as adapting to the communication situation, or talking about places. The communication between a person and a route planner is further challenged because of the different fabric of internal spatial representations and reasoning in persons' minds $[10,44]$ and in machines [39]. With different spatial concepts being involved, ontological mismatches will be expected in the communication of routes.

However, Turing held a philosophical interest on the nature of artificial intelligence. His test does not facilitate a real measure of intelligence of programs, nor does it provide an objective way of testing. For example, when comparing two spatial descriptions it is sometimes hard to say which one is more anthropomorphic. In this sense, we will be able to identify gaps leading to failure of the spatial Turing test, and based on these gaps develop requirements of a conceptual model of a route planner overcoming these gaps. But we will not be able to measure the quality of the communication with a route planner. This means, following Popper's research paradigm [30] the goal for this paper is to refute that machines cannot think spatially (by closing the gaps), but not to prove that machines can think spatially (i.e., prove that all gaps are closed).

\section{Talking to a route planner}

The communication between a wayfinder and a route planning service has the same structure of route communication between two persons. It consists of three phases: the initial phase where the wayfinder asks an informant for directions, the center phase where the informant provides route directions, and the closing phase of confirmations and separation [20,54]. Nearly all research in this area focuses on the center phase [19, $6,34]$.

In this paper the focus is on the initial phase in which the wayfinder has the lead role and talks to the route planner. According to Klein [20, p. 168], the initial phase consists of three subtasks for the wayfinder:

- getting into contact with the informant;

- making clear what he wants;

- succeeding in getting the informant to take over the task of giving him route directions. 
Orthogonally to the temporal sequence of tasks, Klein as well as Wunderlich and Reinelt [54, p. 183] identify three subtasks present at each stage of direction giving. These subtasks are a cognitive task (e.g., activating a spatial cognitive representation); an interactional task (e.g., initiating and terminating the verbal exchange, or providing a route description); and a linguistic task (e.g., expressing a comprehensible route description).

The initial phase was studied neither by Klein nor by Wunderlich and Reinelt in particular, and also other linguistic, cognitive and engineering research focuses rather on human or automatically generated route directions as a whole. However, it is possible to identify in the initial phase already:

- A cognitive task. The wayfinder has to find a proper specification for his route request, which means a specification that is sufficient for the informant in the given communication context. The informant has to activate a spatial cognitive representation and to identify the specification of the wayfinder in this representation.

- An interactional task. The wayfinder has to manage the three subtasks of the initial phase identified above. The informant has to pay attention, listen, and respond by confirming that the specification of a route was received and sufficient.

- A linguistic task. Wayfinder and informant interact via sign systems (a language), and all three subtasks of the initial phase have to be expressed in a sign system and understood by the recipient. The wayfinder has to contact the informant via language, express the request in a language, and be ensured via language that the informant took over.

In this paper the focus is on the interactions between the cognitive and the interactional task, which in the context of a machine as informant includes not only the cognitive abilities of the wayfinder, but also the internal data models and algorithms of the route planner. This means, the focus is on identifying and modeling the references that have to be conveyed and understood, and not on their actual representation in a specific sign system.

Technically, the three subtasks of the initial phase are dependent on the architecture and interface of the route planners. An example can be given for the three web-based route planners of Figure 1. The communication with a web-based planner is realized via the HTTP protocol. Getting into contact is realized by typing a uniform resource identifier in a web browser's address field, or alternatively by following hyperlinks to the route planners' home page (realizing an HTTP get request). Making clear what the wayfinder wants is dictated by the route planners' interface (Figure 1). Succeeding in getting the route planner to take over is realized by pressing a submit button (realizing another HTTP get request, parameterized by form data), and can prolong if there are ambiguities in the request to be resolved.

As outlined in Section 1 a machine, to pass the spatial Turing test, has to understand a wayfinder's request as another person would do. It was also argued above that a machine needs to have properties of an intelligent agent to do so. Hence, a route planner needs to be able to cope with situatedness, embodiment and emergence [1] as experienced by the wayfinder:

- Situatedness: the route planner should be aware of the context of the communication situation. This concerns in particular the location of the wayfinder and the time of the request. 
- Embodiment: the route planner should be aware of the human intuitions underlying their ability to move and their common-sense or naïve understanding of the world [10]. This concerns in particular relative, qualitative and egocentric spatial expressions.

- Emergence: the route planner should be aware of the coherent cognitive structures of the wayfinder evolved during the process of learning spatial environments. This concerns their procedural and declarative spatial knowledge, in particular the hierarchic organization of spatial cognitive representations.

The three elements will be used to structure the requirements analysis in the next section. To be precise, Brooks lists a fourth property of an intelligent agent, intelligence. In the present paper intelligence is seen emerging from the other three elements, applying here Turing's sense to the word, with no further need of a different definition and special requirements.

\section{Requirements analysis}

What are the requirements of a route planner to pass the spatial Turing test with its initial communication phase? When Egenhofer identified database requirements for vehicle navigation in geographic space [9] he identified properties of data and operations wayfinders will want to perform. This wayfinder-centered approach will be continued here. This approach does not start from existing database properties or constraints, but from the behavior of wayfinders when making clear what they want, i.e., specifying a route request.

\subsection{Situatedness}

Each communication takes place in a situation, which forms the context of the communication. The context provides the multiple perspectives needed for understanding the discourse. While people are good at capturing the context, for machines such as route planning services this is a challenge. This section develops a conceptual model of the context of route communication.

According to a classification by Janelle [18], spatial constraints of a communication situation are either physical co-presence or telepresence, while temporal constraints are either synchronous or asynchronous communication. The cases are discussed in Table 1.

Table 1. Janelle's spatial and temporal communication constraints [18] applied on seeking route advice.

\begin{tabular}{|l|l|l|}
\hline & synchronous & asynchronous \\
\hline $\begin{array}{l}\text { physical } \\
\text { co-presence }\end{array}$ & $\begin{array}{l}\text { e.g., face-to-face, or from mobile } \\
\text { location-aware device }\end{array}$ & $\begin{array}{l}\text { e.g., from you-are-here maps, or de- } \\
\text { parture plans at bus stops }\end{array}$ \\
\hline telepresence & $\begin{array}{l}\text { e.g., via telephone or from web ser- } \\
\text { vice }\end{array}$ & e.g., departure plan from a web page \\
\hline
\end{tabular}


Interestingly, in the context of seeking route advice this categorization is insufficient. To capture the full spatiotemporal communication context one needs also to consider the spatiotemporal constraints of the activity the communication is about: wayfinding. One of the two spatial constraints of this activity- the point of departurecan be the current location of the wayfinder (from here), but the communication can also concern a future activity with any other point of departure (from elsewhere). The other spatial constraint of the activity, the desired destination, is independent from any communication context, hence, needs to be specified in any case, and is excluded here from consideration. The temporal constraint of the activity-the time of departure-can be the actual time of the communication (now), but it can also be for any departure in the future (at a later time). These constraints are not completely orthogonal; if a request is for now it is necessarily for here (but not vice versa). Also, sometimes it is not the time of departure but the time of arrival that shall be specified. This case is equivalent to any departure in the future since it is not specific about the time of departure. Together these dimensions span a four-dimensional space (compared to the two-dimensional space of Table 1), with two categories each:

- location of the communicators: physical co-presence or telepresence

- time of the communication: synchronous or asynchronous

- point of departure: from here or from elsewhere

- time of departure: now or at a later time

Let us call any four-tuple of these parameters the context of the communication. Some typical projections of this space to a specific context are:

- <physical co-presence, synchronous, from here, now >:

- A person asking a passer-by: "Can you tell me the way to the city hall?"

- A person studying a bus stop's real-time arrival and departure display.

- A tourist using a mobile location-aware device as a guide for a city tour.

- <physical co-presence, asynchronous, from here, at a later time >:

- A car driver is following the signs to the airport.

- A traveler studying a train station's departure plan.

It is left open whether all 16 possible combinations of context parameters have a reflection in communication scenarios; the observation of a correlation between now and here runs counter such an expectation. The latter case indicates already that the perceived or assumed context is not necessarily identical for the wayfinder and a service. Some specific context mismatches are for example:

- A public transport user arriving at a tram stop is searching for the next tram going to city (<physical co-presence, synchronous, from here, now $>$ ) and finds a plan of departure times (<physical co-presence, asynchronous, from here, at a later time $>$ ).

- A public transport user, just before leaving home, is turning to his desktop for advice on a trip across the city (<telepresence, synchronous, from here, now $>$ ) and finds a service interface asking for a departure location and time $(<\ldots$, from elsewhere, at a later time $>$ ). 
A clear condition for a service to pass the spatial Turing test is to be able to adapt to the context of the wayfinder to an extent any person being asked for route advice would do (see the example above, or $[20,54,14]$ ). Thus, the first postulated requirements are:

1. A service should be accessible anywhere, anytime [49].

When a wayfinding problem arises persons can not solve on their own, they will ask other persons around for advice. A mobile device or a terminal has the same affordance. While the wayfinder does not care whether a service is running locally (physical co-presence) or centrally (telepresence), the wayfinder needs local access, and in case of centralized service architectures this means ubiquitous connectivity.

2. A service should be able to anticipate the spatiotemporal activity context from the communication situation.

If a communication situation suggests a request for from here, this context specification from here should be inferred automatically. Face-to-face communication between people (physical co-presence) does without explicit communication of from here. In contrast, any telepresence communication situation between people will make wayfinders specify their current location on their own initiative. The problem is that while a wayfinder calling a person knows that this other person has no access to the caller's current location and adapts to this situation, this wayfinder cannot recognize whether a service accessible from a local device is running locally (physical co-presence) or centralized (telepresence). Hence, the situation's affordance does not trigger wayfinders to reveal their current location.

Furthermore, communication between people does without explicit communication of now if it can be concluded from the communication situation. Services should be able to behave similarly.

3. A service should be able to understand the human cognitive concepts that are used in the communication about the activity context. This concerns in particular the concepts of from here and now, but also from elsewhere and at a later time.

Route planning services take various inputs, including origin, destination and time of travel, to produce first a route and then a route description. This input needs to be understood by the service such that it can derive a complete parametrization for the optimal route algorithm. But this input should also have anthropomorphic properties to pass a spatial Turing test, i.e., it should allow wayfinders to communicate in their terms. And it is the services's obligation to do the transfer.

The third requirement has consequences for embodiment and emergence, and hence, the discussion re-appears in the following Sections 4.2 and 4.3.

\subsection{Embodiment}

Embodiment in this context postulates a route planner to consider that the wayfinder is a subject having and using a body for movement, and these sensorimotor skills and experiences shape the person's spatial and temporal cognitive concepts. In the center of the initial phase of communication is the specification of a change of place by the wayfinder. The route planning service, to pass a spatial Turing test, should allow 
wayfinders to communicate their specification in their spatial and temporal terms. Besides of considering the situation (Section 4.1), this means to accept and interpret the spatial and temporal references in the verbal expression of the wayfinder.

People answer where questions in a hierarchic manner, either zooming in or zooming out $[37,38,28,29]$. "San Francisco is in the USA, in California." - "My office is in the Department of Geomatics, which is part of the School of Engineering at the university." - "The newspaper is on the table in the living room." - Place descriptions of this form are a special case of general referring expressions [3]. They are not only hierarchically organized, they are also finite (and even relatively short) despite the infinite possibilities to describe a place. A speaker includes just the references that are sufficient in the given communication context. This context is defined by the location where the communication takes place (here) and the place to be described. In fact, initializing the route communication is one form of answering where questions ("Where are you?", "Where do you want to go?").

If the location of the communication is close to the location to be described, references to more global places are neglected, and references to more detailed places are added:

- Conversation at home: "The newspaper is on the table in the living room [of our apartment, which is in Melbourne, Australia]."

- Conversation in a coffee shop: "I left the newspaper [in Australia, in Melbourne,] at home [in the living room, on the table].")

Similarly, but more on a geographic scale:

- Conversation overseas: "I am living in Melbourne, Australia."

- Conversation when entering a taxi at Melbourne airport: "To North Melbourne, near the hospitals, off Flemington road."

- Conversation when friends are ringing the intercom: "Third floor, the apartment opposite the lift."

For the speaker different references appear to be relevant in different contexts. Applying relevance theory [51] we can already determine automatically which references are relevant, based on the distance of references in a hierarchical conceptualization of the environment [26, 27, 4, 46]. Current route planners do not. First, they have a prescriptive and static interface. Secondly, if toponym resolution leads into ambiguities (Melbourne in Australia or in Florida, USA?) some planners always insist that the wayfinder resolves the ambiguity, while other ones at least interpret the destination location in the context of the given start location (if the first address is in Melbourne, Australia, the second address is assumed by default to be in Melbourne, Australia, even if the same street name exist in Melbourne, Florida).

What has been discussed for space so far extends to time in the same manner. Temporal references have a granularity ("tomorrow", "tomorrow afternoon", "tomorrow at $2 \mathrm{pm}$ ", "tomorrow at 2:18pm"), and which granularity is relevant is determined by the communication context.

Furthermore, place descriptions come with spatial prepositions denoting spatial relations between subject and object (see the examples above). Understanding spatial 
prepositions is a challenge for natural language understanding [42], and current web services-route planners as well as local search-interpret only the most basic spatial relationship: in, ignoring all others (except a couple of services interpreting the binary relationship corner). Understanding is impeded by the context dependency and vagueness of qualitative relationships such as near [8].

The second set of requirements, extracted from this discussion, is therefore:

4. A service should always be aware of the current location of the wayfinder, enabling the service for accepting deictic references.

5. A service should apply concepts of relevance to the route specification.

6. A service should have access to hierarchic representations of space and time, and be able to navigate in these representations.

7. A service should have capabilities to understand spatial prepositions and relations.

Related to the embodied experience of space and time is the structure of the immediate environment of the wayfinder. However, this aspect is covered by the discussion of emergence in the next section.

\subsection{Emergence}

Emergence in complex systems refers to the way patterns at macroscopic levels arise out of a large number of interactions at microscopic levels. In the current context the relevant complex system is the procedural and declarative knowledge of humans of their environment. It was mentioned already that cognitive spatial representations have a hierarchical order. Accordingly, human reasoning for route planning is hierarchically organized, which has inspired hierarchical route planning strategies [43, 50]. Movement is planned at higher levels first, and then filled with actions at lower levels (down to the subconscious level of body coordination during locomotion). Movement specifications, as they contain the description of the destination, can be hierarchic as well (see Section 4.2).

An aspect not discussed before are the structures and constraints of the environment of the wayfinder at different levels of these hierarchies. Of central interest are the human concepts of here and now, both having vague and context-dependent semantics. Here and now may be references to places and times over some levels of granularity, and at each level place and time may not be crisply defined. The point of departure being here can mean the actual position of the wayfinder, but also generalized concepts of place, or the nearest access to a transportation network. Similarly, the departure now can mean immediate departure but also departure with the next available transportation means, or the one after if its more convenient.

Imagine planning to travel from Melbourne to Tokyo. Current route planners are based on the airline transportation networks ${ }^{2}$. Automatically they identify places such as Melbourne or Tokyo with airports, as airports represent the nodes in these transportation networks. They expect that the wayfinder is able to find the way to the airport in Melbourne on their own, or alternatively chooses another route planner for this part of

\footnotetext{
${ }^{2}$ For example, http: //www. quantas.com or http://www. expedia.com.
} 
the travel. The public transport planner for Melbourne ${ }^{3}$ might help here. It guides the wayfinder from a nearby tram stop to the airport, using the airport bus. The bus arrives in front of the arrival hall, but the planner tells the wayfinder that from the arrival stop a walk of $230 \mathrm{~m}$ leads to Melbourne Airport. Whatever the granularity of this metric detail is, this destination is not the wayfinder's check-in counter. Thus, the wayfinder needs another route planner for finding the way through the airport. This time she might choose a display board of departures, or the information booth, or a You-Are-Here map. To find one of these she has to make route planning decisions on her own, based on perceived affordances. - Combining these individual route planning processes to one, one finds a recursively refining specification of the route: "I want to go [from here] to Tokyo." - "Ok, but tell me how to find to the airport." - "And at the airport, where is the check-in counter for the flight to Tokyo?" And so on. These multiple hierarchies in route planning and communication were studied, for example, by Rüetschi and Timpf [36]. Current route planners fail to realize this properly.

Now let us assume that the wayfinder has specified in all cases the time of departure as now (or a time in the future). The airline route planner knows only that the wayfinder is in Melbourne. The public transport planner ignores that she is at home at the time of the request (or she ignores to specify in her request that she is currently at home). Only the display board of departures at the tram stop assumes that the wayfinder is within eyespot (strictest physical co-presence). With other words, the notions of here and now have different meanings in different communication contexts (again, this context is determined by the current location in comparison to the destination, which varies here between Tokyo, the airport and the check-in counter).

Thus, the following requirements can be derived from the discussion:

8. A service should capture the spatiotemporal constraints of the route to resolve the vagueness of wayfinder-defined terms here and now.

9. A service should apply means of time geography [25] at all levels of spatial and temporal granularity to deal with the inherent vagueness of here and now.

10. A service should, therefore, acknowledge that all route planning is inherently timedependent. People may give different route directions by day than by night, or at peak hours or non-peak hours.

11. A service should care for integrated route planning on different transportation networks (of different scales and different means of transportation).

In principle, also the spatial and temporal constraints of the communication come with vaguely defined categories. Physical co-presence may extend, for example, to earshot or eyespot, and telepresence as its counterpart has complementary vague meaning. Synchronous communication may accept some delay such as we experienced in the early days of Skype ${ }^{4}$. However, the communication context is not verbally communicated. Thus the task of understanding and mapping human notions is limited to the spatiotemporal constraints of the activity, here and now.

\footnotetext{
${ }^{3}$ http: //metlinkmelbourne.com.au.

${ }^{4}$ Skype is a registered trademark.
} 


\section{Comparison of the requirements with current route planning services}

In this micro-cosmos of a person talking to a route planner at least two ontologies (or epistemologies, to be precise) $[40,13]$ meet: the human wayfinder's ontology of the environment and the service's internal ontology. Talking to a route planner a wayfinder provides place descriptions, at least for the destination, and potentially a time description. These descriptions have to be mapped by the route planning service to its internal ontology. Essentially this forms a problem of understanding place descriptions.

The service's internal ontology is defined by graph theory and network analysis. To determine a route it needs to translate any input to two unambiguous positions on a transportation network, the start $s^{\prime}$ and the destination $d^{\prime}$. This process of translation may include toponym identification and resolution, georeferencing the toponyms by looking up gazetteers [41], map matching to draw the georeference to the next point on the transportation network, and dynamic segmentation of the transportation network to include temporarily these two points to the network for a shortest path algorithm. Additionally, for time-dependent shortest path problems one of both times is required: the departure time or the arrival time $t^{\prime}$. This internal ontology of a service, $\left(s^{\prime}, d^{\prime}, t^{\prime}\right)$, shows in any current route planner interface (e.g., Figure 1). These interfaces are static and do not adopt to the situation, except that they may fill the form fields with default values.

In current route planners this translation has restricted functionality. While gazetteers help to resolve toponyms, current gazetteers contain only authoritative geographic placenames [16]. Other databases may add privately collected placenames such as the entries in Yellow Pages, or the stop names of a public transport provider. However, all these resources do not yet contain synonyms, vernacular placenames or common abbreviations of placenames. They also do not contain information about the spatial extent of a place, or of its relationships with other places. Hence, route planners are typically structuring and limiting the input by a wayfinder. Furthermore, most if not all spatial prepositions and relationships cannot be resolved and are ignored.

In comparison the wayfinder ontology is loosely captured by the requirements listed in Section 4. The 11 requirements go beyond the capabilities of current route planners. To prove the hypothesis we have to demonstrate that these requirements demand fundamental changes to the interface as well as the internal functionality of current route planners.

In this respect, not all requirements have the same standing. Some requirements look at fundamental change for some route planners. An example is R10 ("Acknowledge that all route planning is inherently time-dependent"). Typical route planning services for walking, biking or car navigation do not consider time. To match better anthropomorphic behavior they should. But the knowledge to realize these fundamental changes exists, and some services comply already, such as vehicle navigation systems linking with traffic message channel. Thus, requirements of this sort do not prove the hypothesis.

But other requirements stand out. The most challenging ones may be R3, R6 and R7, or understanding the human concepts of here and now, hierarchical place descriptions, and spatial prepositions. All three of them are in conflict with current interfaces as 
well as with current capacities of language understanding. At least with these three requirements the hypothesis is proven.

\section{Conclusions}

This paper studies the initial phase of the communication between a human wayfinder and a route planning service. It starts from requirements derived by a vision of a service of anthropomorphic understanding and behavior. These requirements are fundamentally different from the ontologies currently implemented, and hence, require a new approach to accept and understand wayfinders' expressions about their spatial and temporal travel constraints. In particular we argue that a service needs to be able to understand place descriptions, human concepts of here and now, and the spatial prepositions in natural language expressions.

The approach to derive these requirements was based on the argument that a route planning service deals with an autonomous and mobile physical agent, and hence, has to reflect the elements of this agent: being situated, embodied and capable of dealing with a complex geographic environment. Although this approach is systematic, no claims are made to be complete. The identified requirements will close some gaps on the way to a service that would pass a spatial Turing test.

Some of the remaining open questions concern:

- The construction of cognitively motivated hierarchic structures of spatial representations. To reflect the cognitive representations of people of their environment, these hierarchies should contain synonyms, elements of vernacular geography (where boundaries are often different to authoritative boundaries) and for all elements of a city [23]. Accordingly, toponyms should be represented by their spatial extent, and this requires a capacity to represent and deal with vague concepts. Furthermore, the order in the hierarchy should be determined by cognitively salient characteristics. Some research in this direction has started [47, 52], but a consistent, comprehensive and integrated model for all elements of a city or any other environment is missing.

- Free-form place descriptions consist not only of hierarchically ordered toponyms but also of spatial prepositions that need to be interpreted to fully capture the sense of a place description. While the hierarchy represents part-of relationships, other spatial relationships between geographic features need to be represented as well, implicitly or explicitly. Some of them are already captured in spatial databases, for example on connectivity and topology. But since they are not (yet) available in gazetteers, methods to exploit them for understanding place descriptions are lacking. What is also lacking are models for the interpretation of relations of vague or context-dependent meaning, such as nearness [8]. Going further, Kuhn postulates research in cognitive semantic engineering [22]. The latter establishes models capturing the semantics of spatial concepts, acknowledging that perception (which informs human conceptualization) is situated and embodied, and Gestalt is emerging from complex perceptive and cognitive processes in a complex spatial environment.

- Since understanding place descriptions is a special case of natural language understanding, the vertical and horizontal relationships captured before have to be applied for the understanding of human place descriptions. While the preliminary 
hierarchies are successfully used to automatically generate place descriptions [35, 46], the reverse process of understanding given place descriptions is not yet investigated. Again, an area where we can profit from current research is the interpretation of sketches in graphical query interfaces to geographic information systems [11]. Although in this particular case the sign system is different from natural ${ }^{5}$ language, the subject is the same: communication about place.

Furthermore, within the given context of a route communication we so far expected descriptions of enduring places in geographic space, such that toponyms can be stored in databases. However, more transient cases of place exist as well, and some of them can even have a meaning in route communication. A person may, for example, look for the way to a meeting. And metaphorical places (e.g., heaven) will further be excluded.

\section{Acknowledgments}

Part of this work is supported under the Australian Research Council's Discovery Projects funding scheme (project number 0878119).

\section{References}

1. Brooks, R.A.: Intelligence without reason. In Myopoulos, J., Reiter, R., eds.: 12th International Joint Conference on Artificial Intelligence IJCAI-91, San Mateo, CA, Morgan Kaufmann Publishers (1991) 569-595

2. Burnett, G.E.: "Turn right at the traffic lights": The requirement for landmarks in vehicle navigation systems. The Journal of Navigation 53(3) (2000) 499-510

3. Dale, R.: Generating Referring Expressions: Constructing Descriptions in a Domain of Objects and Processes. MIT Press, Cambridge, Mass. (1992)

4. Dale, R., Geldof, S., Prost, J.P.: Using Natural Language Generation in Automatic Route Description. Journal of Research and Practice in Information Technology 37(1) (2005) 89 105

5. Daniel, M.P., Tom, A., Manghi, E., Denis, M.: Testing the value of route directions through navigational performance. Spatial Cognition and Computation 3(4) (2004) 269-289

6. Denis, M.: The description of routes: A cognitive approach to the production of spatial discourse. Current Psychology of Cognition 16(4) (1997) 409-458

7. Denis, M., Pazzaglia, F., Cornoldi, C., Bertolo, L.: Spatial Discourse and Navigation: An Analysis of Route Directions in the City of Venice. Applied Cognitive Psychology 13 (1999) $145-174$

8. Duckham, M., Worboys, M.: Computational Structure in Three-Valued Nearness Relations. In Montello, D.R., ed.: Spatial Information Theory. Volume 2205 of Lecture Notes in Computer Science. Springer, Berlin (2001) 76-91

9. Egenhofer, M.: What's special about spatial? Database requirements for vehicle navigation in geographic space. In: ACM SIGMOD International Conference on Management of Data, Washington, ACM Press (1993) 398-402

\footnotetext{
${ }^{5}$ Although this might be the correct technical term to characterize spoken or written discourse, one could argue that drawing sketches is also a natural way of expression. It is probably even the older way to communicate than by words.
} 
10. Egenhofer, M.J., Mark, D.M.: Naive Geography. In Frank, A.U., Kuhn, W., eds.: Spatial Information Theory. Volume 988 of Lecture Notes in Computer Science. Springer, Berlin (1995) 1-15

11. Egenhofer, M.J.: Query Processing in Spatial-Query-by-Sketch. Journal of Visual Languages and Computing 8(4) (1997) 403-424

12. Elias, B., Sester, M.: Incorporating landmarks with quality measures in routing procedures. In Raubal, M., Miller, H.J., Frank, A.U., Goodchild, M.F., eds.: Geographic Information Science. Volume 4197 of Lecture Notes in Computer Science. Springer, Berlin (2006) 6580

13. Frank, A.U.: Ontology. In Kemp, K.K., ed.: Encyclopedia of Geographic Information Science. Sage Publications, Thousand Oaks, CA (2007)

14. Freundschuh, S.M., Mark, D.M., Gopal, S., Gould, M.D., Couclelis, H.: Verbal Directions for Wayfinding: Implications for Navigation and Geographic Information and Analysis Systems. In Brassel, K., Kishimoto, H., eds.: 4th International Symposium on Spatial Data Handling, Zurich, Department of Geography, University of Zurich (1990) 478-487

15. Hansen, S., Richter, K.F., Klippel, A.: Landmarks in OpenLS: A Data Structure for Cognitive Ergonomic Route Directions. In Raubal, M., Miller, H.J., Frank, A.U., Goodchild, M.F., eds.: Geographic Information Science. Volume 4197 of Lecture Notes in Computer Science. Springer, Berlin (2006) 128-144

16. Hill, L.L.: Georeferencing: The Geographic Associations of Information. Digital Libraries and Electronic Publishing. The MIT Press, Cambridge, MA (2006)

17. Hirtle, S.C., Jonides, J.: Evidence of hierarchies in cognitive maps. Memory and Cognition 13(3) (1985) 208-217

18. Janelle, D.G.: Impact of information technologies. In Hanson, S., Giuliano, G., eds.: The Geography of Urban Transportation. Guilford Press, New York (2004) 86-112

19. Klein, W.: Wegauskünfte. Zeitschrift für Literaturwissenschaft und Linguistik 33 (1979) 9-57

20. Klein, W.: Local Deixis in Route Directions. In Jarvella, R.J., Klein, W., eds.: Speech, Place, and Action. John Wiley \& Sons, Chichester (1982) 161-182

21. Klippel, A., Tappe, H., Habel, C.: Pictorial representations of routes: Chunking route segments during comprehension. In Freksa, C., Brauer, W., Habel, C., Wender, K.F., eds.: Spatial Cognition III. Volume 2685. Springer, Berlin (2003) 11-33

22. Kuhn, W.: Why Information Science needs Cognitive Semantics. In: Workshop on the Potential of Cognitive Semantics for Ontologies (FOIS 2004), Torino, Italy (2004)

23. Lynch, K.: The Image of the City. MIT Press, Cambridge (1960)

24. Maaß, W.: Von visuellen Daten zu inkrementellen Wegbeschreibungen in dreidimensionalen Umgebungen: Das Modell eines kognitiven Agenten. Phd thesis, Universität des Saarlandes (1996)

25. Miller, H.J.: Activities in Space and Time. In Hensher, D.A., Button, K.J., Haynes, K.E., Stopher, P.R., eds.: Handbook of Transport Geography and Spatial Systems. Volume 5 of Handbooks in Transport. Elsevier, Amsterdam (2004) 647-660

26. Paraboni, I., Deemter, K.v.: Generating Easy References: The Case of Document Deixis. In: Second International Conference on Natural Language Generation (INLG 2002), New York, USA (2002) 113-119

27. Paraboni, I., Deemter, K.v., Masthoff, J.: Generating referring expressions: Making referents easy to identify. Computational Linguistics 33(2) (2007) 229-254

28. Plumert, J.M., Carswell, C., DeVet, K., Ihrig, D.: The Content and Organization of Communication about Object Locations. Journal of Memory and Language 34 (1995) 477-498

29. Plumert, J.M., Spalding, T.L., Nichols-Whitehead, P.: Preferences for ascending and descending hierarchical organization in spatial communication. Memory and Cognition 29(2) (2001) 274-284 
30. Popper, K.: The Logic of Scientific Discovery. Routledge Classics. Routledge, London (2002)

31. Raubal, M., Winter, S.: Enriching Wayfinding Instructions with Local Landmarks. In Egenhofer, M.J., Mark, D.M., eds.: Geographic Information Science. Volume 2478 of Lecture Notes in Computer Science. Springer, Berlin (2002) 243-259

32. Richter, K.F., Klippel, A.: A Model for Context-Specific Route Directions. In Freksa, C., Nebel, B., Knauff, M., Krieg-Brückner, B., eds.: Spatial Cognition IV. Volume 3343 of Lecture Notes in Artificial Intelligence. Springer, Berlin (2005) 58-78

33. Richter, K.F., Klippel, A.: Before or after: Prepositions in spatially constrained systems. In Barkowsky, T., Knauff, M., Ligozat, G., Montello, D.R., eds.: Spatial Cognition V. Volume 4387 of Lecture Notes in Artificial Intelligence. Springer, Berlin (2007) 453-469

34. Richter, K.F.: Context-Specific Route Directions. Volume 3 of Monograph Series of the Transregional Collaborative Research Center SFB/TR8. Akademische Verlagsgesellschaft, Berlin (2008)

35. Richter, K.F., Tomko, M., Winter, S.: A dialog-driven process of generating route directions. Computers, Environment and Urban Systems 32(3) (2008) 233-245

36. Rüetschi, U.J., Timpf, S.: Modelling wayfinding in public transport: Network space and scene space. In Freksa, C., Knauff, M., Krieg-Brückner, B., Nebel, B., Barkowsky, T., eds.: Spatial Cognition IV: Reasoning, Action, and Interaction. Volume 3343 of Lecture Notes in Artificial Intelligence. Springer, Berlin (2005) 24-41

37. Shanon, B.: Where Questions. http://acl.ldc.upenn.edu//P/P79/ P79-1017.pdf (1979)

38. Shanon, B.: Answers to Where-Questions. Discourse Processes 6 (1983) 319-352

39. Shekhar, S., Vatsavai, R.R., Ma, X., Yoo, J.S.: Navigation systems: A spatial database perspective. In Schiller, J., Voisard, A., eds.: Location-Based Services. Morgan Kaufmann Pubblishers, San Francisco, CA (2004) 41-80

40. Smith, B.: Ontology and information science. In Zalta, E.N., Nodelman, U., Allen, C., eds.: Stanford Encyclopedia of Philosophy. Center for the Study of Language and Information, Stanford University, Stanford, CA (2003) (online)

41. Stokes, N., Li, Y., Moffat, A., Rong, J.: An empirical study of the effects of NLP components on geographic IR performance. International Journal of Geographical Information Science 22(3) (2008) 247-264

42. Tenbrink, T.: Space, Time, and the Use of Language: An Investigation of Relationships. Mouton de Gruyter, Berlin (2007)

43. Timpf, S., Volta, G.S., Pollock, D.W., Frank, A.U., Egenhofer, M.J.: A conceptual model of wayfinding using multiple levels of abstraction. In Frank, A.U., Campari, I., Formentini, U., eds.: Theories and Methods of Spatio-Temporal Reasoning in Geographic Space. Volume 639 of Lecture Notes in Computer Science. Springer, Berlin (1992) 348-367

44. Timpf, S.: Ontologies of wayfinding: A traveler's perspective. Networks and Spatial Economics 2(1) (2002) 9-33

45. Tom, A., Denis, M.: Referring to landmark or street information in route directions: What difference does it make? In Kuhn, W., Worboys, M., Timpf, S., eds.: Spatial information theory. Volume 2825 of Lecture Notes in Computer Science. Springer, Berlin (2003) 384 397

46. Tomko, M., Winter, S.: Pragmatic construction of destination descriptions for urban environments. Spatial Cognition and Computation, accepted for publication on 21 August 2008 (2008)

47. Tomko, M., Winter, S., Claramunt, C.: Experiential hierarchies of streets. Computers, Environment and Urban Systems 32(1) (2008) 41-52

48. Turing, A.M.: Computing machinery and intelligence. Mind 59(236) (1950) 433-460 
49. Weiser, M.: The Computer for the Twenty-First Century. Scientific American (9) (1991) 94-104

50. Wiener, J.M., Mallot, H.A.: 'Fine-to-coarse' route planning and navigation in regionalized environments. Spatial Cognition and Computation 3(4) (2003) 331-358

51. Wilson, D., Sperber, D.: Relevance Theory. In Horn, L.R., Ward, G., eds.: Handbook of Pragmatics. Blackwell, Oxford (2004) 607-632

52. Winter, S., Tomko, M., Elias, B., Sester, M.: Landmark hierarchies in context. Environment and Planning B 35(3) (2008) 381-398

53. Winter, S., Wu, Y.: The "spatial Turing test". In Navratil, G., ed.: Colloquium for Andrew U. Frank's 60th Birthday. Volume 39 of Geoinfo Series., Vienna, Austria, Department for Geoinformation and Cartography, Technical University Vienna (2008) 109-116

54. Wunderlich, D., Reinelt, R.: How to get there from here. In Jarvella, R.J., Klein, W., eds.: Speech, Place, and Action. John Wiley \& Sons, Chichester (1982) 183-201 